\title{
Emotion and the brain: multiple roads are better than one
}

\author{
Luiz Pessoa* and Ralph Adolphs ${ }^{\ddagger}$
}

We have recently provided a critical evaluation of the idea that a subcortical pathway through the superior colliculus and pulvinar to the amygdala has a prominent and privileged role in processing affective visual stimuli (Emotion processing and the amygdala: from a 'low road' to 'many roads' of evaluating biological significance. Nature Rev. Neurosci. 11, 773-783 (2010)) ${ }^{1}$. In so doing, one of our goals was to encourage a careful re-evaluation of the purported subcortical pathway. We therefore welcome the correspondence by de Gelder and colleagues (Emotion and the brain: of low roads, high roads, and of royal roads less traveled. Nature Rev. Neurosci. 15 June 2011 (doi:10.1038/nrn2920-c1) ${ }^{2}$, in which they argue for the importance of this subcortical pathway. As we have already addressed at length the points that they raise in our original paper, we will focus on three overarching issues.

\section{Multiple visual pathways}

In our Perspective', we outlined a 'multiple roads' framework in which the processing fate of biologically relevant stimuli is better understood in terms of 'multiple roads' that lead to the expression of observed behaviours, rather than a dichotomy between a 'low road' versus a 'high road'. Accordingly, we reviewed anatomical evidence that supports the existence of multiple 'bypass pathways' that rapidly convey emotion-laden information throughout the brain. The proposal of multiple visual processing pathways is nothing new, of course ${ }^{3}$.

However, the past few years have been particularly exciting in demonstrating additional visual pathways that bypass the lateral geniculate nucleus (LGN), as well as pathways that involve the LGN but bypass the early visual cortex. For example, Wurtz and colleagues ${ }^{4}$ have described not only one but multiple LGN-bypassing visual pathways, including a pathway from the superior colliculus to the frontal eye fields through the medial dorsal thalamus, and two pathways from the superior colliculus to the middle temporal area (area MT) in visual cortex via the pulvinar. These pathways are involved in multiple aspects of 'active vision', including saccade- and attention-related processes. Recent studies combining lesions and functional $\mathrm{MRI}^{5}$ are particularly exciting in advancing our understanding of the parts played by these 'non-standard' visual pathways.

\section{Effect of amygdala lesions}

In our Perspective ${ }^{1}$, we discussed evidence from studies of patients with amygdala lesions, which permit causal inferences that are not possible with many other methods, such as fMRI. As we discussed, a recent study ${ }^{6}$ showed that reaction times for detecting fearful faces in a patient with complete bilateral amygdala lesions were normal, as was the ability of fearful facial expressions to break into conscious perception during binocular suppression. These findings reveal that the amygdala cannot be essential for non-conscious, rapid fear detection (at least in the tasks used in the study) but, as pointed out by de Gelder et al. ${ }^{2}$, they are limited to one case study. However, recent patient studies support and extend these initial findings.

One such study assessed visual search in patients with unilateral amygdala lesions ${ }^{7}$ and found that they detected emotional targets more efficiently than neutral targets, and at the same level as control participants. A second study asked patients with unilateral amygdala lesions to complete an attentional task in which emotional distractors impaired the perception of subsequent targets; again, the group showed completely normal effects (R. M. Piech, M. McHugo, S. D. Smith, M. S. Dukic, J. Van Der Meer, B. Abou-Khalil, S. B. Most and D. H. Zald, personal communication). Even more conclusively, a third study revealed that two individuals with selective bilateral amygdala lesions exhibited normal (enhanced) detection of aversive words during the attentional blink ${ }^{8}$. Taken together, the findings from lesion studies demonstrate that the amygdala is not a necessary component of several of the functions traditionally attributed to the 'low road'.

\section{Role of the cortex and subcortical structures}

De Gelder et al. ${ }^{2}$ suggest that our framework returns to a corticocentric view of emotion processing. Far from it, our Perspective $^{1}$ devoted considerable attention to the physiology and anatomy of subcortical structures such as the pulvinar. Although we urged a re-evaluation of the existence of an uninterrupted subcortical visual pathway (the evidence does not favour such a view), we were also concerned to emphasize the important parts that are played by subcortical structures such as the amygdala and pulvinar.

The medial pulvinar, in particular, is bidirectionally connected to multiple areas involved in assessing the biological significance of an item, including parietal, cingulate, insular and orbitofrontal cortices. Interestingly, both the amygdala and the pulvinar have been suggested to be part of an 'innermost core' network of brain regions that is topologically central ${ }^{9}$ - that is, strongly connected to all other regions of the core and the rest of the brain. Related to this, the role of thalamic nuclei in regulating information transfer between cortical regions - that is, cortico-thalamo-cortical communication is increasingly appreciated ${ }^{10}$.

We are thus in agreement with de Gelder et al. ${ }^{2}$ that subcortical structures have key roles in emotion processing. Where we differ is in doubting the anatomical existence of a straight subcortical route to the amygdala and in doubting the functions traditionally attributed to such a putative route. We do believe that subcortical structures, like the amygdala and the pulvinar (as well as other subcortical nuclei), need to be incorporated into network models of emotion processing. However, their functions will make sense only when they are considered as being embedded in circuits that also encompass multiple cortical regions. We favour neither a subcorticocentric nor a corticocentric view of emotion processing, but one in which the vertical and horizontal integration of information is central.

*Department of Psychology, Neuroscience and Cognitive Science Program, University of Maryland, College Park, Maryland 20742, USA.

${ }^{\ddagger}$ Division of Humanities, Social Sciences, Computation and Neural Systems Program, California Institute of Technology, Pasadena, California 91125, USA.

e-mails:pessoa@umd.edu; radolphs@hss.caltech.edu doi:10.1038/nrn2920-c2

1. Pessoa, L. $₫$ Adolphs, R. Emotion processing: from a 'low road' to 'many roads' of evaluating biological significance. Nature Rev. Neurosci. 11, 773-783 (2010).

2. de Gelder, B., van Honk, J. \& Tamietto, M. Emotion in the brain: of low roads, high roads and roads less travelled. Nature Rev. Neurosci. 15 Jun 2011 (doi:10.1038/nrn2920-c1).

3. Ungerleider, L. G. \& Mishkin, M. Analysis of Visual Behavior (eds Ingle, D. J., Goodale, M. A. \& Mansfield, R. J. W.) 549-586 (MIT Press, Cambridge, 1982). 
CORRESPONDENCE

4. Wurtz, R. H., McAlonan, K., Cavanaugh, J. \& Berman, R. A. Thalamic pathways for active vision. Trends Cogn Sci. 15, 177-184 (2011)

5. Schmid, M. C. et al. Blindsight depends on the lateral geniculate nucleus. Nature 466, 373-377 (2010).

6. Tsuchiya, N., Moradi, F., Felsen, C., Yamazaki, M. \& Adolphs, R. Intact rapid detection of fearful faces in the absence of the amygdala. Nature Neurosci. 12 ,

1224-1225 (2009).

7. Piech, R. M. et al. Fear-enhanced visual search persists after amygdala lesions. Neuropsychologia 48, 3430-3435 (2010).

8. Bach, D. R., Talmi, D., Hurlemann, R., Patin, A. \&

Dolan, R. J. Automatic relevance detection in the absence of a functional amygdala. Neuropsychologia 49, 1302-1305 (2011).

9. Modha, D. S. \& Singh, R. Network architecture of the long-distance pathways in the macaque brain. Proc Natl Acad. Sci. USA 107, 13485-13490 (2010).

10. Theyel, B. B., Llano, D. A. \& Sherman, S. M. The corticothalamocortical circuit drives higher-order cortex in the mouse. Nature Neurosci. 13, 84-88 (2010). 